\title{
Formation Mechanism of Fibrous Silica from a Suspension of Monodispersed $\mathrm{SiO}_{2}$
}

\author{
Hiroyuki NAKAMURA, Akira SHIMIZU* and Yoko SUYAMA* \\ Inorganic Materials Department, Kyushu National Industrial Research Institute, AIST, 807-1, Shuku-machi, Tosu-shi, Saga 841-0052 \\ *Department of Material Science, Shimane University, 1060, Nishikawazu, Matsue-shi, Shimane690-8504
}

\author{
単分散シリカ懸濁液からの繊維状シリカの生成メカニズム \\ 中村浩之·清水 啓*·陶山容子* \\ 九州工業技術研究所ファイン素材部，841-0052 佐賀県鳥栖市宿町 807-1 \\ *島根大学総合理工学部物質科学科, 690-8504 島根県松汇市西川津町 1060
}

\begin{abstract}
Fibrous silica was prepared by drying a homogeneous suspension of monodispersed silica particles. The silica fibers consisted of almost closely packed particles. In this study, silica fibers as long as $7 \mathrm{~cm}$ and $40-200 \mu \mathrm{m}$ in width were obtained. The main driving force for fiber generation is considered to be the tensile stress which is generated by the water content distribution. The fiber forms in the following steps: (1) formation of closely packed homogeneous $\mathrm{SiO}_{2}$ film; (2) generation of cracks by shrinking of the film; and, (3) formation of fibers by propagation of the cracks. The fiber width was large when the silica film was thick, the silica particles were large, and the drying temperature was low. Silica fibers were not obtained when the suspension $\mathrm{pH}$ was as low as 0.5 , the suspension did not wet the substrate well and the silica film was not thick enough. The prepared fiber was very fragile, however, it can be strengthened by calcination. The fiber's have ca. $20 \mathrm{~nm}$ homogeneous pores after calcining at $800^{\circ} \mathrm{C}$ for $1 \mathrm{~h}$.
\end{abstract}

[Received June 21, 2000; Accepted November 13, 2000]

Key-words : Porous fiber, Silica, Pore size distribution, Morphology, Colloidal crystal, Monodispersed particles, Drying

\section{Introduction}

In a fixed bed catalytic reactor, a granulated particle is often used for easy handling. In this case, a granulated particle with a low pressure loss is often desired for the effective use of the catalyst surface. When the pressure loss at the particles is high, it is difficult for a reactant to diffuse into the granulated particles and thus the effective surface area decreases. From the viewpoint of the diffusion coefficient, a large diameter pore such like a meso-size pore is preferred. The preparation of meso-porous materials was often performed by utilizing a liquid crystal template, ${ }^{1), 2)}$ colloidal crystal $^{3), 4)}$ and so on. In addition, if it is possible to make a fiber from meso-porous materials, reactants can easily flow through the gap between the fibers, and the effective surface area would geometrically increase, because the thickness of the fibers is smaller than the diameter of spheres with the same volume.

Many types of ceramic fibers such as $\mathrm{SiO}_{2}, \mathrm{TiO}_{2}, \mathrm{Al}_{2} \mathrm{O}_{3}$, $\mathrm{BaTiO}_{3}$ have been fabricated by the sol-gel process, $\left.{ }^{5}\right)$ drawing from a viscous sol following sintering. This is a common method, however, the prepared fibers are usually dense. On the other hand, Jung et al., fabricated zeolite fibers by a unique method. ${ }^{6), 7)}$ They dried a suspension of monodispersed nano-order zeolite particles in a test tube and obtained a porous fiber. During the drying, the zeolite particles in the suspension were deposited on the wall of the test tube to make a two-dimensionally developed homogeneous film of zeolite particles. As the drying proceeded, the silica film had many cracks running from the top to the bottom of the tube that generated the zeolite fibers. Using this method, they obtained zeolite fibers with a $2.2 \mathrm{~mm}$ average length and 50-70 aspect ratio. However, the formation mechanism of the fibers is still not sufficiently clear, and applicability of this method to materials other than the MFI-type zeolites is not known.

In this study, the fabrication of silica fibers was investigated using a method employed by Jung et al. ${ }^{6), 7)}$ The fiber formation process was observed and the mechanism is discussed. Furthermore, the effect of the preparation conditions on the morphology of the fibers such as the width and thickness were investigated.

\section{Experiments}

Silica particles were prepared by the Stober method. ${ }^{8)}$ An aqueous ammonium solution was added to the ethanol solution in which TEOS was previously dissolved. The mixed solution was agitated for about one minute and stored for $1 \mathrm{~d}$. The obtained turbid solution was centrifuged to correct the deposited silica particles. The corrected particles were re-dispersed in $\mathrm{pH} 1 \mathrm{HNO}_{3}$ solution to remove the ammonium ions and washed with distilled water until the $\mathrm{pH}$ of the solution became 5-6. Finally, the solution was condensed to about 10-15 mass \% and stocked. In this study, two kinds of silica particles, $0.32 \mu \mathrm{m}$ ( $C V$ (coefficient of variation $)=$ $12 \%)$ and $0.16 \mu \mathrm{m}(C V=18 \%)$ were prepared and used. The stocked suspension was diluted with distilled water to $0.13-2.0$ mass $\%$ and ultra-sonicated. The suspension was then dried at $90-110^{\circ} \mathrm{C}$ in a drying oven. Some of the fibers obtained were calcined at 800 or $1000^{\circ} \mathrm{C}$ for $1 \mathrm{~h}$ in air.

A borosilicate glass test tube (inner diameter $=1.5 \mathrm{~mm}$ ), the polypropyrene test tube (inner diameter $=1.5 \mathrm{~mm}$ ) or a polystyrene bottle was used as a drying vessel. When a suspension was dried in a polystyrene bottle, a slide glass (borosilicate glass) was set in the bottle as schematically shown in Fig. 1. Just before the experiments, the glass tubes and the slide glasses were carefully washed with $1 \mathrm{~N}$ $\mathrm{NaOH}$ and thoroughly rinsed.

Fibers obtained from the drying equipment shown in Fig. 1 were observed using a microscope to determine the fiber width distributions. In the drying experiments using the equipment shown in Fig. 1, fibrous silica was obtained only on the up-side of the slide glass, but no fibers were obtained on the polypropylene bottle wall and down-side of the slide glass. From the fibers generated on the slide glass, 

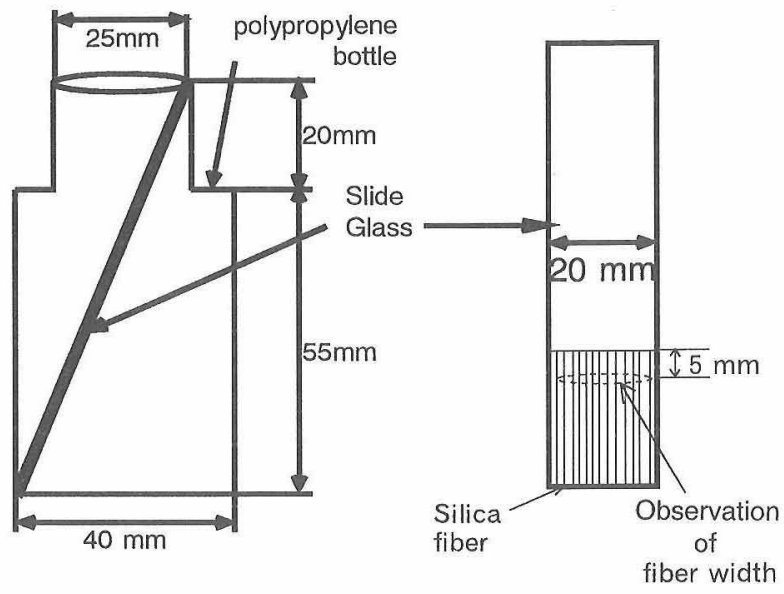

Fig. 1. Drying vessel.

about 200 fibers were randomly chosen. The width of each fiber was measured at a point $5 \mathrm{~mm}$ from the top of the silica film. The thickness of some of the fibers was also measured by the optical microscope. The SEM observation was performed to determine the particle diameter distribution and to observe the surface morphology of the fibers. To elucidate the fiber generation mechanism, the crack growth process was observed using a video recorder equipped with a microscope. The specific surface area and pore size distribution of the fibers after calcination were measured with a mercury porosimeter.

\section{Results and discussion}

3.1 Preparation of silica fibers from a silica suspension Figure 2 shows the silica fibers prepared in a test tube using a 1 mass $\%$ suspension of $160 \mathrm{~nm}$ silica particles. The fibrous silica shown in Fig. 2(A) was easily obtained by drying the suspension at $100^{\circ} \mathrm{C}$. The fiber length was about $7 \mathrm{~cm}$ and the color was white. An optical micrograph of the fiber is shown in Fig. 2(B). The fibers have a spline-like shape and they were transparent in the thickness direction. The surface morphology of a fiber is shown in Fig. 2(C). It was obvious that the fiber was consisted of almost close packed silica particles. The surface morphology of the silica fiber showed no obvious difference with that of the zeolite fibers reported by Jung et al. ${ }^{6), 7)}$ The $\mathrm{SiO}_{2}$ particles consisting of fibers did not re-disperse by just immersing and stirring in water.

During drying, the silica fibers continuously formed in the vicinity of the meniscus of the dispersion. Figure 3 shows a photograph of the vicinity of the meniscus during drying. From this figure, it is obvious that a silica fiber was made by cracking of the silica film formed on the substrate. For the $\mathrm{SiO}_{2}$ particle film formation from a suspension, Jiang et al. also recently investigated drying the suspension at room temperature. ${ }^{9)}$ They reported that the particles in the film were 3-dimensional closely packed and particles of homogeneous diameter were necessary to obtain a defectless particle layer. They considered the mechanism of the film formation to be basically the same as that of the monoparticle film formation reported by Nagayama et al. ${ }^{10)-12)}$

3.2 Effect of wettability of suspension to wall

When a polypropylene tube was used as the drying vessel, the silica film was hardly formed on the tube wall and the fibrous silica was not obtained. On the other hand, when ethanol, which had a good wettability with the polypropylene wall, was used as the dispersive medium, the silica film was formed on the wall and the fibrous silica was obtained
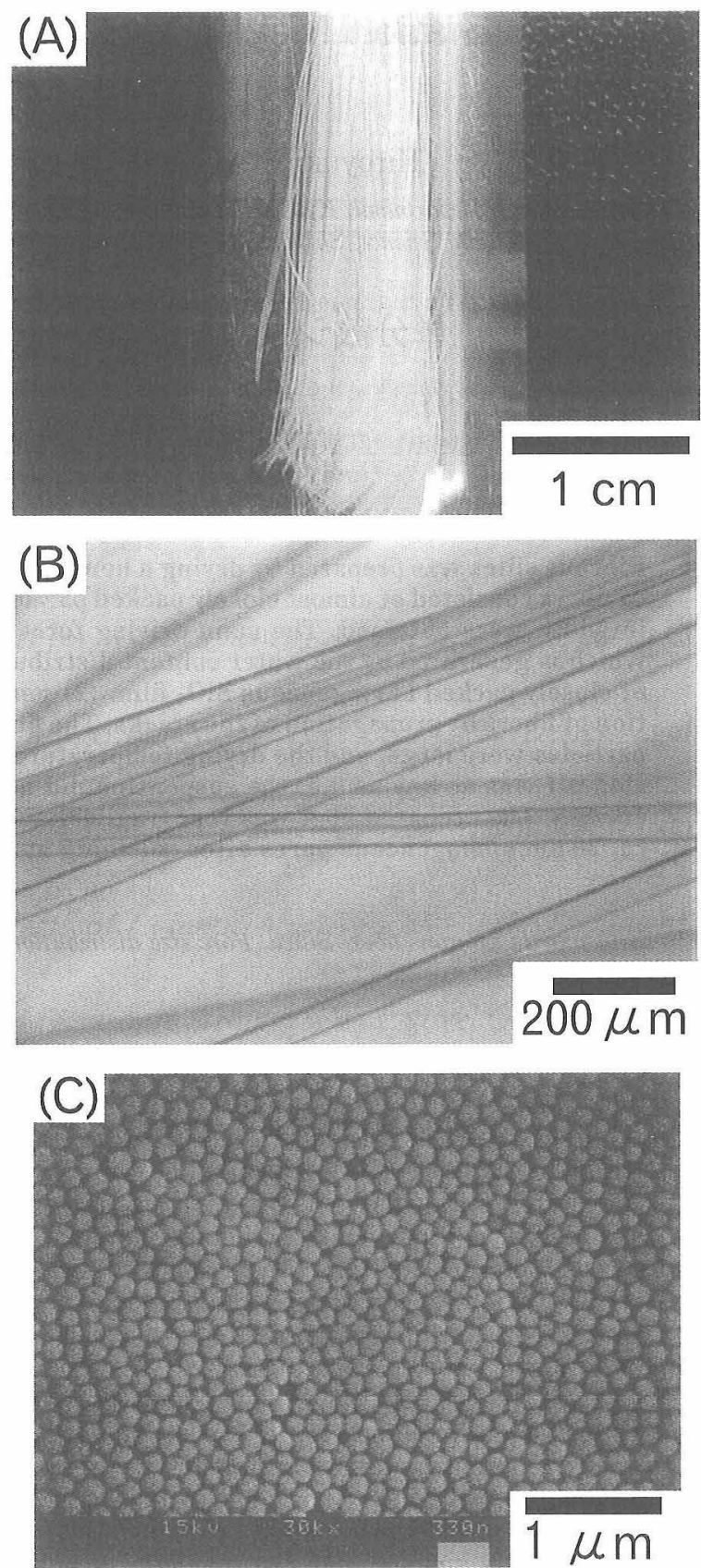

Fig. 2. Morphology of fibrous silica.

$\mathrm{SiO}_{2}$ diameter $=0.16 \mu \mathrm{m}, \mathrm{SiO}_{2}$ concentration $=1.0$ mass $\%$, drying temperature $=100^{\circ} \mathrm{C}$, dried in a test tube $(D=15 \mathrm{~mm})$.

(A) Photograph, (B) optical micrograph and (C) SEM micrograph.

These results show the importance of wetting between the suspension and substrate. Denkov et al. discussed the preparation process of two-dimensional colloidal crystals on a substrate. They mentioned that their two dimensional monolayer colloidal crystals were prepared by nucleus formation governed by an attractive capillary force and following convective particle flux caused by the water evaporation from the already ordered array. ${ }^{11)}$ When the thickness of the meniscus was large, the capillary force was not occur and the particles layer was not originated.12) This can explain the reason why we cannot obtain particle layers on the polypropylene wall. 


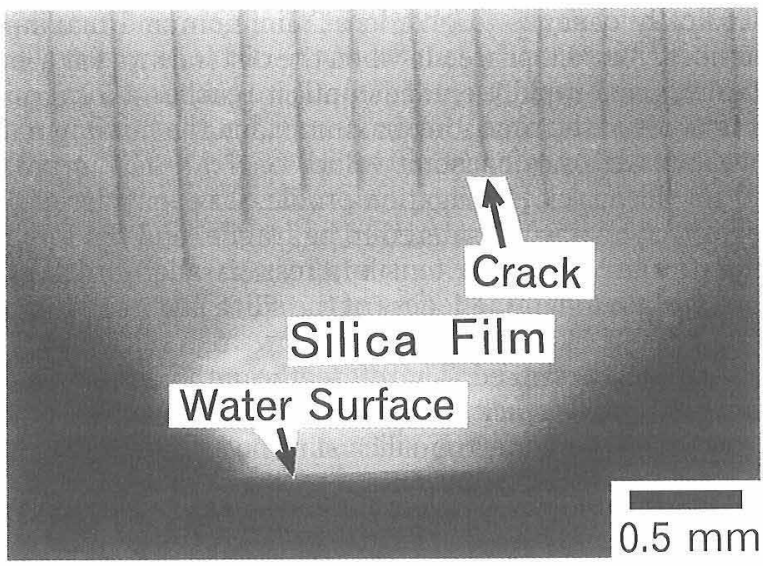

Fig. 3. Vicinity of water surface during drying.

$\mathrm{SiO}_{2}$ diameter $=0.16 \mu \mathrm{m}, \mathrm{SiO}_{2}$ concentration $=2.0$ mass $\%$, drying temperature $=100^{\circ} \mathrm{C}$, dried in a test tube $(D=15 \mathrm{~mm})$.

3.3 Effect of suspension $\mathrm{pH}$ on the fiber morphology The effect of the suspension $\mathrm{pH}$ on fiber morphology was investigated. The $\mathrm{pH}$ value of the suspensions were adjusted to $5.0,2.0,1.0$ and 0.5 by nitric acid. The suspension $\mathrm{pH}$ without adjustment was 5.0 and its conductivity was $8 \times$ $10^{-6} \Omega / \mathrm{cm}$, which was the same order as that of distilled water used in this experiment $\left(2 \times 10^{-6} \Omega / \mathrm{cm}\right)$ showing a considerably low ionic strength. A measurement of the $\zeta$ potential revealed that the pzc (point of zero charge) of the silica particles used in this study was about 0.8 .

Suspensions with various $\mathrm{pHs}$ were dried in the test tubes. The surface morphology of the products is shown in Fig. 4. From the suspensions of $\mathrm{pH}=5.0$ and 2.0, fibrous silica was obtained. On the other hand, no fibrous silica was obtained from the suspension of $\mathrm{pH}=0.5$. From the $\mathrm{pH}=$ 1.0 suspension, fibrous silica was observed on the upper part of the film, however, their width rapidly increased, and finally, the visible cracks disappeared to make a continuous film of silica.

In the figure, silica particles are almost close-packing for the case of $\mathrm{pH}=5$. The silica fiber from the $\mathrm{pH}=2.0$ suspension had almost the same morphology. On the other hand, for the case of $\mathrm{pH}=0.5$, silica particles were loosely packed and many fine cracks were observed in the film. When the $\mathrm{pH}$ of the suspension was 1.0, the upper part where the fibers were observed was similar to the $\mathrm{pH}=5.0$ case, and the lower part where the fibers were not generated was similar to the case of $\mathrm{pH}=0.5$.

The loose and inhomogeneous packing is considered to produce many large voids and small cracks that reduce the tensile stress which occurs by drying and not generating large cracks to form fibers. From this result, the existence of tensile stress as a main driving force for crack generation is considered, and it is also shown that a homogeneous silica
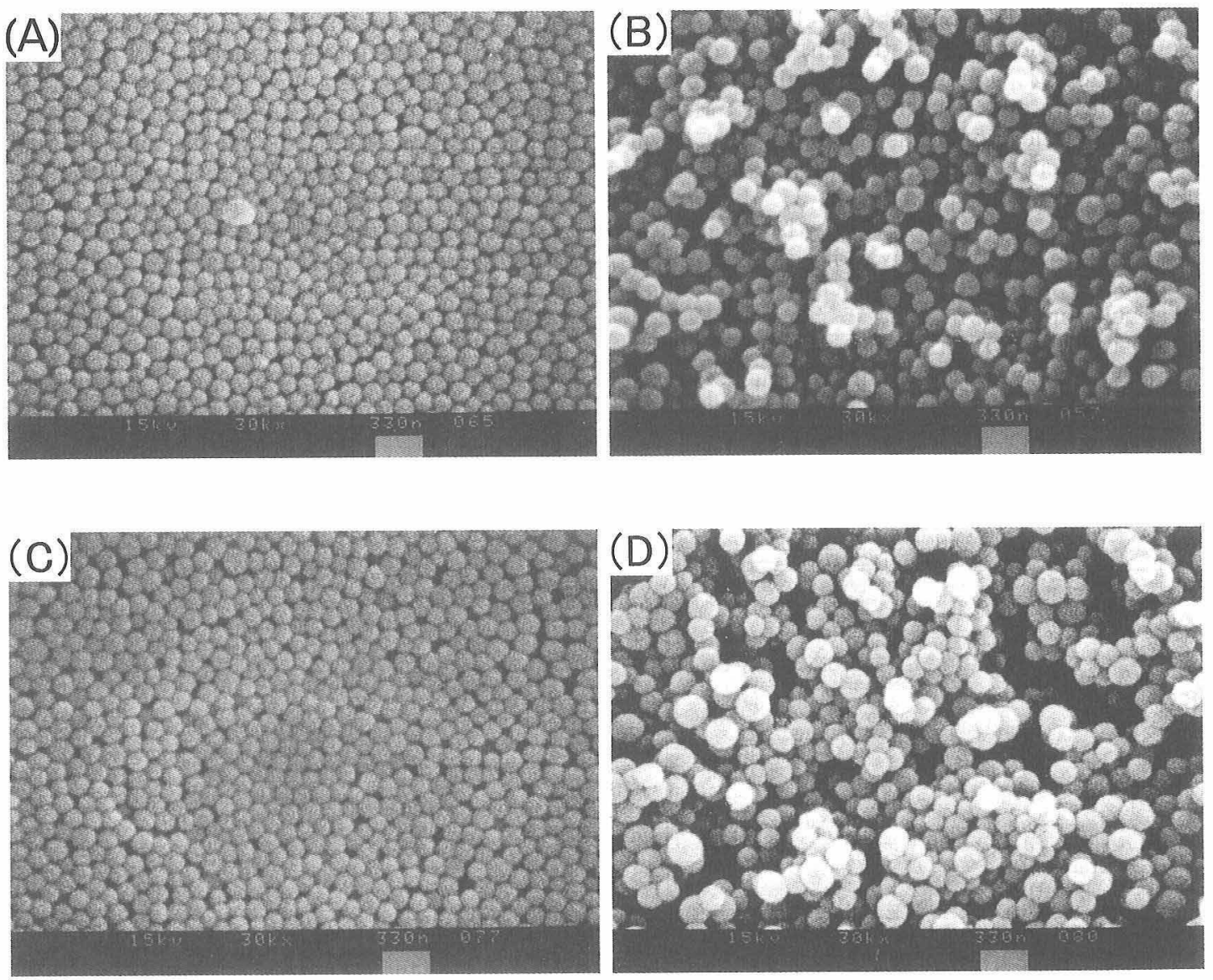

\section{$0.5 \mu \mathrm{m}$}

Fig. 4. Effect of dispersion $\mathrm{pH}$ on fiber morphology.

(A) $\mathrm{pH} 5.0$, (B) $\mathrm{pH} 0.5$, (C) $\mathrm{pH} 1.0$ upper part and (D) $\mathrm{pH} 1.0$ lower part. 
film is necessary in order to make cracks to generate fibers.

The reasons for the effect of the suspension $\mathrm{pH}$ on the packing state of particles are considered as follows. In the case of $\mathrm{pH}=5$, the surface potential was high and the ionic strength was low thereby creating a large repulsive force between particles. When the $\mathrm{pH}$ was 0.5 , the suspension was not stable because of the low surface potentials of the particles (iep $=0.8$ ) and the high ionic strength of the solution. In this suspension, flocculation during drying was observed. Because such aggregates were deposited on the glass wall, the particles were not able to be regularly packed but make an inhomogeneous film. In the case of $\mathrm{pH}=1.0$, the colloids were more stable than the $\mathrm{pH}=0.5$ suspension due to the lower ionic strength. In this case, the ions dissolved in the water were condensed by water vaporization to increase the ionic strength. Sedimentation of such aggregated particles may also produce an inhomogeneous film. Therefore, it is considered that fibrous silica was obtained on the upper wall and not on the lower wall.

3.4 Fiber generation mechanism

At first, in order to investigate the driving force of the crack propagation, the effect of the substrate shape on the direction of fiber generation was investigated. When a cylindrical borosilicate tube (a cut test tube) was stood in a silica suspension and dried, the fibers are generated both of the convex and concave side of the tube. Moreover, even when the cut test tube was laid in the bottom of the suspension, the fibers were generated in the vertical direction to the water surface regardless the change in the direction of curvature. On the other hand, an SEM observation of the crack edge revealed that there are no remarkable relationships between the direction of crack propagation and particle alignment. These results showed that the direction of fiber generation (i.e., crack propagation) was not affected by the curvature of the substrate surface and particle alignment in the film, but always vertical to the suspension surface. From these results, the existence of some force that make a crack vertical to the water level was considered.

Next, in order to observe the crack growth process, a 2 mass \% suspension of $0.16 \mu \mathrm{m}$ silica was dried in a test tube and movements of the water level and crack tips were monitored. These results are shown in Fig. 5. The observation was started after the crack growth became stable. From the figure, it is noticeable that the water level continuously went down while cracks periodically extended. As the result, the distance between the crack tip and water surface also

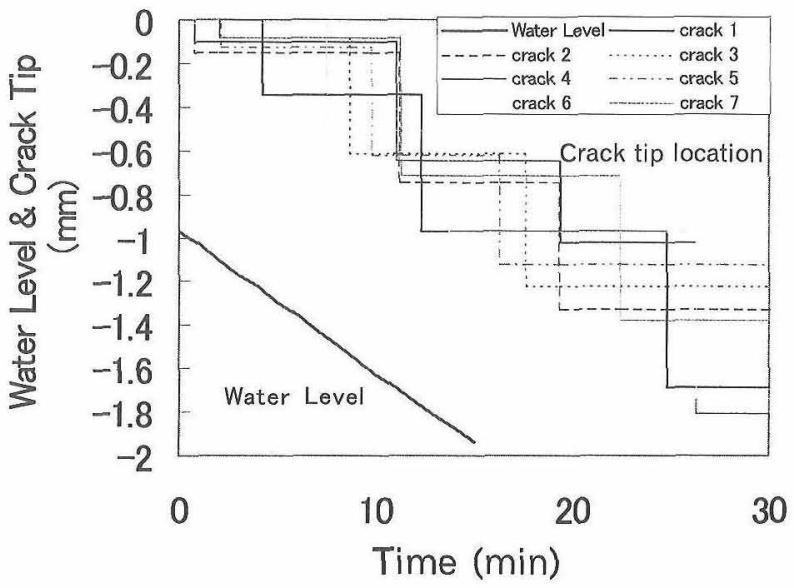

Fig. 5. Change in crack tip location and water surface during drying.

Preparation conditions: see Fig. 3. periodically changes to give local minimum and maximum distances. A crack proceeds when the distance was at a local maximum and rapidly extends until it reaches a local minimum. Most of the time, the maximum and the minimum distances are almost a constant value.

From this crack propagation profile, it is considered that the $\mathrm{SiO}_{2}$ layer was considered to be fragile, and the force to generate the cracks are tensile stress parallel direction to water surface. The fragileness of the silica layer at the crack tip shows the water content was low and silica particles were hardly rearranged. Therefore, the authors consider the driving force for crack propagation to be shrinkage of the silica particle film. In the silica film on the substrate, the water content distribution in the vicinity of the water surface can be postulated, because the process is basically a drying process (at $100^{\circ} \mathrm{C}$ ) and the silica film is continuously deposited from the suspension surface. A water content distribution will arise in the silica film and it produces a shrinkage distribution in the film that turns into a tensile stress distribution. As the water content distribution is large in the vertical direction to the water surface, the shrinkage distribution is large in the same direction and the direction of the tensile stress will be parallel to the water surface.

Here, we discuss a mechanism of the fiber generation which is consistent with the results. A schematic model of the mechanism is shown in Fig. 6. At first, a homogeneous silica film is deposited on the substrate from the suspension by drying. As discussed above, a water content distribution will arise in the silica film and it produces a shrinkage distribution of the film, that turns to be a tensile stress distribution. As the water content distribution is large in the vertical direction to the water surface, the shrinkage distribution is large in the same direction, and the direction of the tensile stress will be parallel to the water surface. As long as there are no cracks on the film, the tensile stress is larger the far away from the water surface because of water content decreases with the distance from the water surface. As shown in Fig, 5, the water surface moves downward while the crack tip stops, which increases the distance between suspension surface and crack tip, thus increasing the tensile

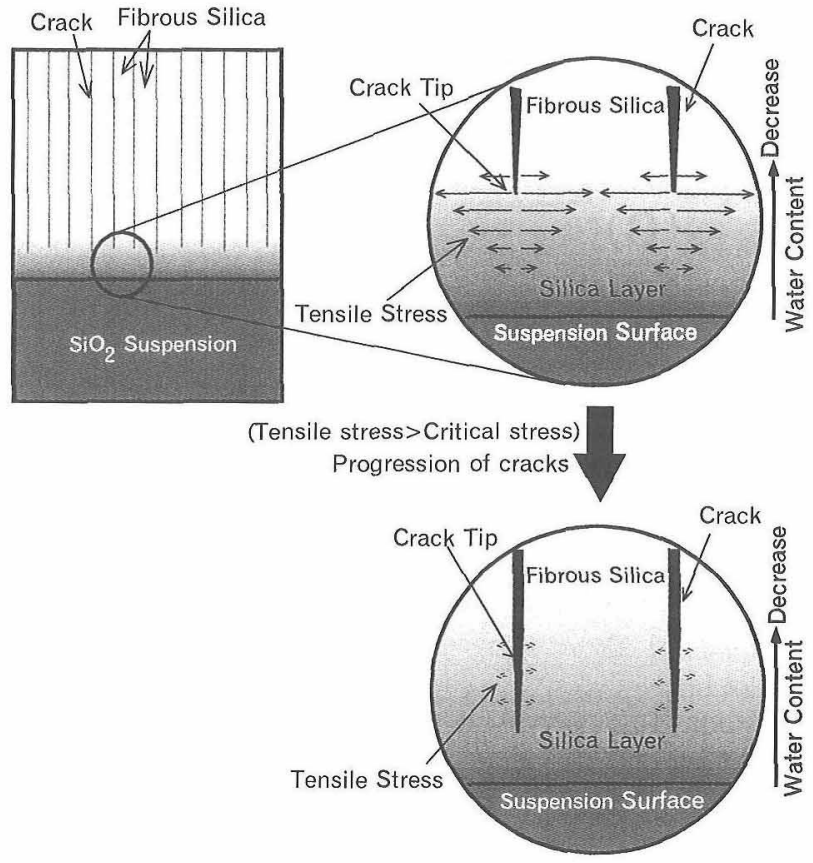

Fig. 6. Schematic representation of crack propagation. 
stress at the crack tip. When the stress at the crack tip exceeds the critical strength of the silica film, the crack propagates until the tensile stress is reduced enough to stop its progress. As the direction of the cracks are always vertical to the water surface in this mechanism, the cracks will propagate in one direction and parallel to each other. The distance between the water surface and crack tip just before the crack propagation will be a maximum distance and the one just after the propagation will be a minimum distance. The maximum and minimum distance should be constant if the film is homogeneous and the strength of the film and water content distribution does not change. After the crack propagation, the water level continuously goes down to make the distance between crack tip and water surface large and the tensile stress at the crack tip increase again. When the stress reaches the critical value, the crack propagates again. The distance between each crack (i.e., fiber width) is considered to be almost the same, when the film is homogeneous.

3.5 Effect of preparation conditions on fiber width distribution

When the fibrous silica was prepared, some preparation conditions affected the fiber width distributions. Therefore, the effects of the experimental conditions on the fiber width distribution were investigated using the drying vessel as shown in Fig. 1. The conditions investigated here were, concentration of suspension (0.13-0.5 mass \%), $\mathrm{SiO}_{2}$ particle size $(0.16$ and $0.32 \mu \mathrm{m})$ and drying temperature $\left(90-110^{\circ} \mathrm{C}\right)$. Figure 7 shows the effect of suspension concentration on the fiber width distribution. The average fiber width was $40 \mu \mathrm{m}$ for the 0.25 mass $\%, 83 \mu \mathrm{m}$ for the 0.38 mass $\%$, and $210 \mu \mathrm{m}$ for the 0.5 mass $\%$ suspensions. From a 0.13 mass $\%$ suspension, no fibers were obtained. From this figure, it is noted that the wider fiber was obtained from the more condensed silica suspension in this region and fibers were not obtained when the suspension was too dilute.

When a fiber was prepared in a test tube, the fiber width became wider in the downward direction. Therefore, a relationship between the fiber width and fiber thickness was investigated. This result is shown in Fig. 8. The width has changed from $50 \mu \mathrm{m}$ to $400 \mu \mathrm{m}$ as the fiber thickness changed from $15 \mu \mathrm{m}$ to $130 \mu \mathrm{m}$, showing that the thicker fiber gives a wider fiber. The effect of the suspension concentration on the fiber width can be attributed to the effect of fiber thickness. The reason for the thicker layer in the lower part is considered to be due to condensation during the drying.

Figure 9 shows the effect of $\mathrm{SiO}_{2}$ particle size on the fiber width distribution. The average width was $40 \mu \mathrm{m}$ and $82 \mu \mathrm{m}$

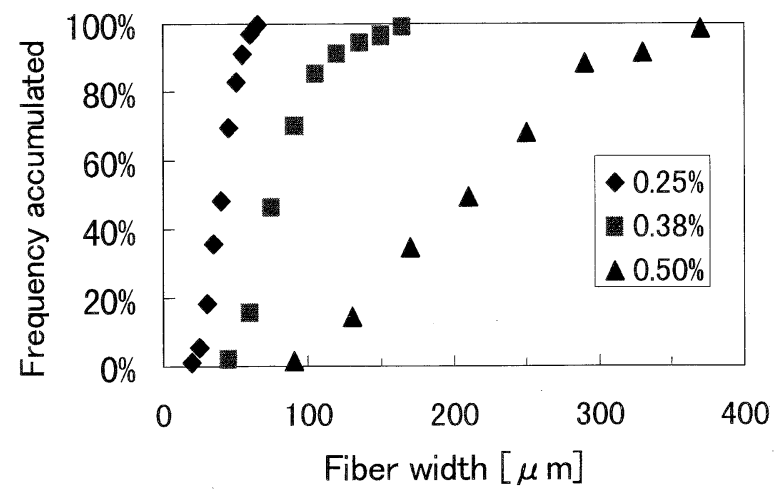

Fig. 7. Effect of suspension concentration on fiber width distribution.

$\mathrm{SiO}_{2}$ diameter $=0.16 \mu \mathrm{m}$, drying temperature $=100^{\circ} \mathrm{C}$ for the $0.16 \mu \mathrm{m}$ and $0.32 \mu \mathrm{m}$ in particle sizes, respectively, showing that a larger particle size gives a larger width in the fiber.

The relationship between drying temperature and fiber width is shown in Fig. 10. The average widths for the drying temperatures of $110^{\circ} \mathrm{C}$ and $100^{\circ} \mathrm{C}$ were $47 \mu \mathrm{m}$ and 53 $\mu \mathrm{m}$, respectively, and showed no obvious difference. On the other hand, in the case of drying at $90^{\circ} \mathrm{C}$, the average width became $81 \mu \mathrm{m}$.

From these results, among these experimental conditions, it was shown that for a high concentration, large $\mathrm{SiO}_{2}$ particles, and a low drying temperature gave wide fibers.

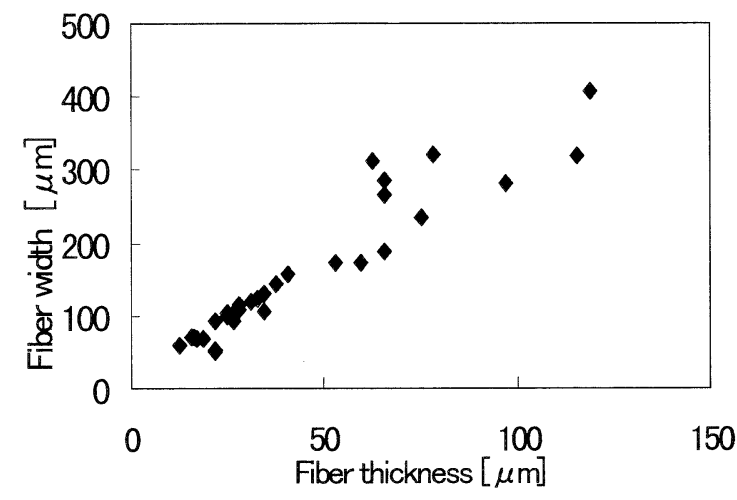

Fig. 8. Relationship between fiber thickness and width. Preparation conditions: see Fig. 2.

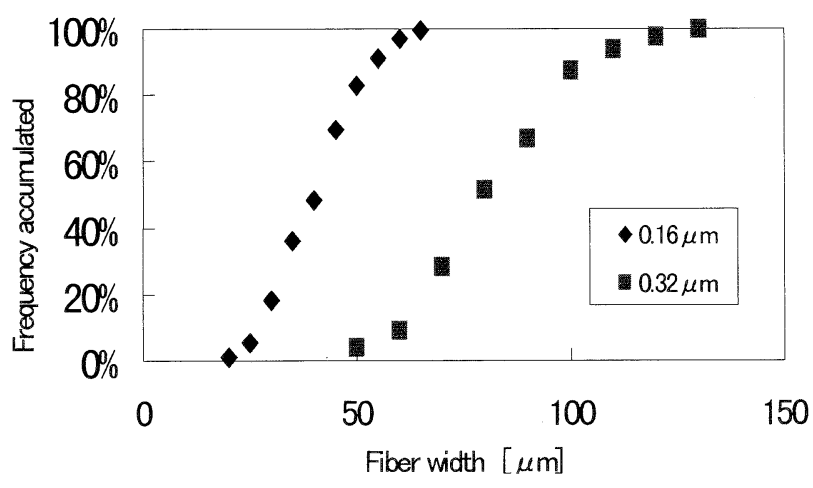

Fig. 9. Effect of particle diameter on fiber width distribution. $\mathrm{SiO}_{2}$ suspension concentration $=0.25$ mass $\%$, drying temperature $=100^{\circ} \mathrm{C}$.

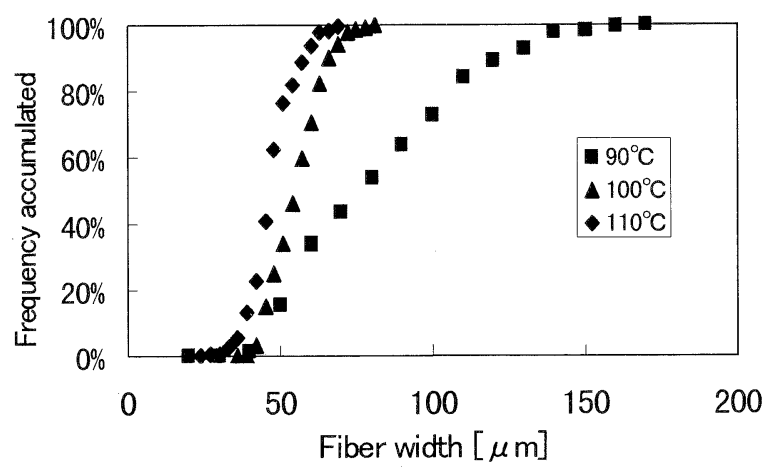

Fig. 10. Effect of drying temperature on fiber width distribution. $\mathrm{SiO}_{2}$ dispersion concentration $=0.25$ mass $\%$, average diameter of $\mathrm{SiO}_{2}=0.16 \mu \mathrm{m}$ 


\subsection{Thermal treatment of fibrous silica}

The prepared silica fibers were so fragile, therefore, it was hard to hold one fiber with tweezers. By calcination at $800^{\circ} \mathrm{C}$ for $1 \mathrm{~h}$, the fibers became much stronger as could be hold by tweezers and slightly bent. The surface morphology of the calcined fibers is shown in Fig. 11. The surface morphology of the fiber after an $800^{\circ} \mathrm{C}, 1 \mathrm{~h}$ calcination showed no distinguished difference with that before calcination. The color of the fiber also remained translucent. When the fiber was calcined at $1000^{\circ} \mathrm{C}$ for $1 \mathrm{~h}$, the color changed to transparent and the pores observed in the $800^{\circ} \mathrm{C}$ calcined fibers disappeared. The strength of the fiber was much stronger as
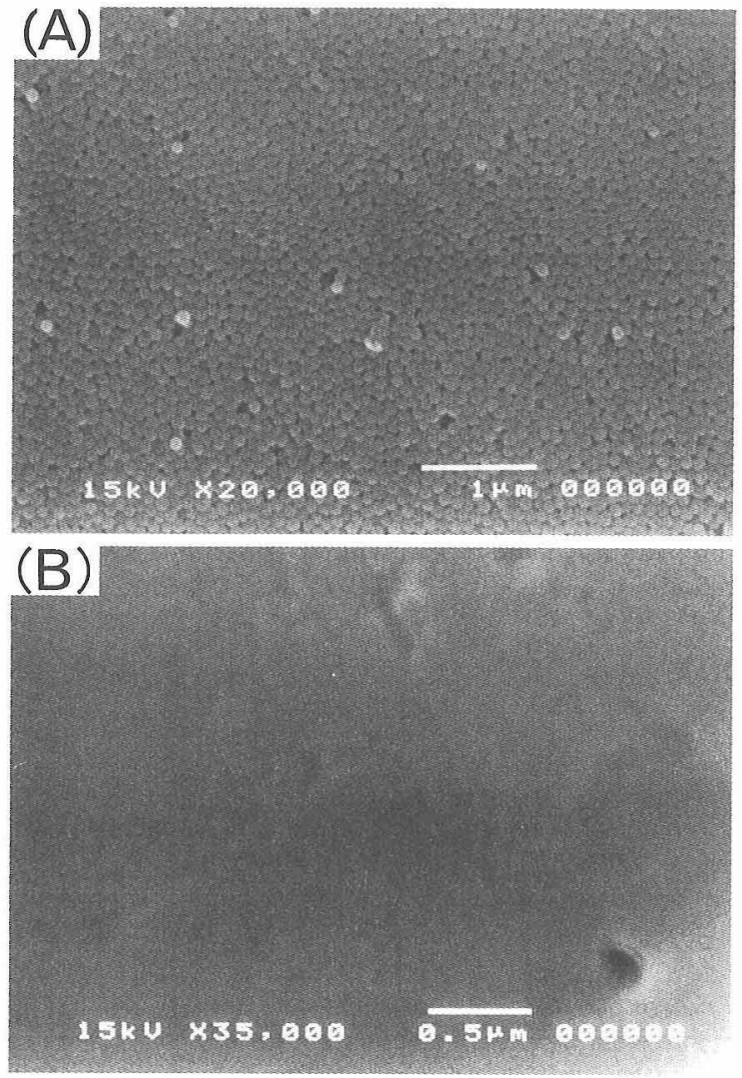

Fig. 11. Surface morphology of calcined fibers. Preparation conditions: see Fig. 2.

(A) $800^{\circ} \mathrm{C}, 1 \mathrm{~h}$ and $(\mathrm{B}) 1000^{\circ} \mathrm{C}, 1 \mathrm{~h}$.

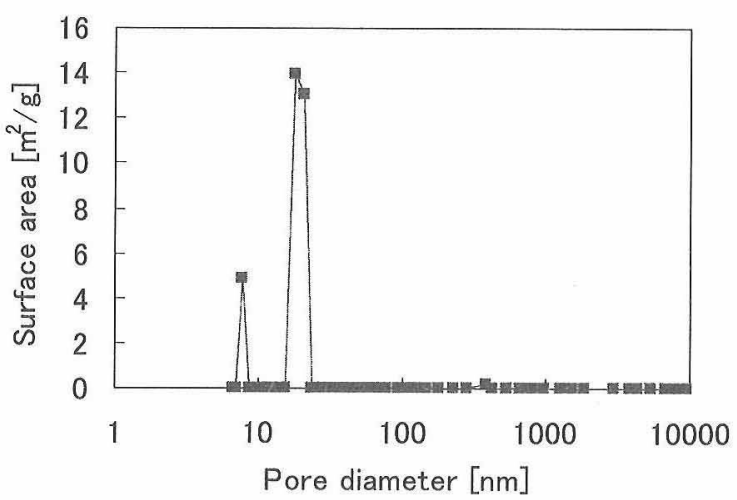

Fig. 12. Pore diameter distribution of silica fiber (calcined at 800 ${ }^{\circ} \mathrm{C}$ for $1 \mathrm{~h}$ ).

Preparation conditions: see Fig. 2. it could be bent with a tweezers.

A pore diameter distribution of the $800^{\circ} \mathrm{C}$ calcined fibers is shown in Fig. 12. From this figure, it is obvious that there is a sharp peak around $20 \mathrm{~nm}$, which roughly corresponds to a diameter of inter-granular pore of the silica particles (24 $\mathrm{nm}$ for $160 \mathrm{~nm}$ silica particle in diameter). The specific surface area of the fiber was $34 \mathrm{~m}^{2} / \mathrm{g}$.

\section{Summary}

Fibrous silica was prepared by drying a silica suspension. Under the experimental conditions, it was possible to prepare a fibrous silica of $7 \mathrm{~cm}$ in length and $40-200 \mu \mathrm{m}$ in width. The surface morphology of the silica fibers consisted of almost close-packed silica particles. When the suspension concentration was low or the contact angle of the meniscus was large, the film thickness was small and no fibers were generated. The direction of the silica fiber generation was always perpendicular to the water level.

The formation mechanism of the fibers was elucidated as follows:

(1) Formation of closely-packed homogeneous $\mathrm{SiO}_{2}$ film.

(2) Generation of cracks by shrinkage of the film.

(3) Formation of fibers by propagation of cracks.

Some experimental conditions affected the fiber width. A concentrated silica solution (i.e., thick film), large silica particles, and low drying temperature gave a wide fiber. The particle diameter and drying temperature also influenced the fiber width. The larger silica particles and the lower drying temperature gave wider fibers.

As prepared fibers were translucent and fragile, however, they became strong by calcination at $800^{\circ} \mathrm{C}$ for $1 \mathrm{~h}$. A measurement of the pore diameter distribution revealed that they have homogeneous pores with a diameter around 20 $\mathrm{nm}$. After calcination at $1000^{\circ} \mathrm{C}$, they became transparent and the strength was much higher than that at RT. The pores observed until at $800^{\circ} \mathrm{C}$ disappeared at $1000^{\circ} \mathrm{C}$.

\section{References}

1) Kresge, C. T., Leonowicz, M. E., Roth, W. J., Vartuli, J. C. and Beck, J. S., Nature, 359, 710-12 (1992).

2) McGrath, K. M., Dabbs, D. M., Yao, N., Aksay, I. A. and Gruner, S. M., Science, 277, 552-55 (1997).

3) Matsushita, S., Miwa, T. and Fujishima, A., Chem. Lett., 1997, 925-26 (1997)

4) Wijnhoven, J. E. G. J. and Vos, W. L., Science, 281, 802-04 (1998).

5) Brinker, C. J. and Scherer, G. W., "Sol-Gel Science," Academic Press, NY (1990).

6) Jung, K. T., Hyun, J. H. and Shul, Y. G., Zeolites, 19, 161-68 (1997).

7) Jung, K. T., Hyun, J. H., Shul, Y. G., Koo, K. K. and Kim, D. S., J. Ceram. Soc. Japan, 106, 1056-61 (1998).

8) Azuma, Y., Tajima, Y., Oshima, N. and Suehiro, K., J. Ceram. Soc. Japan, 94, 559-63 (1986) [in Japanese].

9) Jiang, P., Bertone, J. F., Hwang, K. S. and Colvin, V. L., Chem. Mater., 1999, 2132-40 (1999).

10) Nagayama, K., J. Soc. Powd. Technol., Jpn., 32, 456-85 (1995).

11) Denknov, N. D., Velev, O. V., Kralchevsky, P. A., Ivanov, I. B., Yoshimura, H. and Nagayama, K., Nature, 361, 26 (1993).

12) Dimitrov, A. S. and Nagayama, K., Langmuir, 12, 1303-11 (1996). 